\title{
Effect of defect angle and wavelength on micro-jet forms
}

\author{
L. Chao, W. Pei \& F. Qijing \\ Institute of Applied Physics and Computational Mathematics, China
}

\begin{abstract}
When a shock wave is reflected from a metal surface with groove defects, the micro-jets would be emitted from the free surface of the sample. The mass, velocity, and distribution of the micro-jet depends not only on the shock wave conditions and material properties of the metal, but also on the shape and wavelength of the defects on the sample surface. To understand the effect of the groove angle and wavelength on the micro-jet properties, an elastic plastic hydrodynamics Euler code was applied. The maximum velocity, ejecting factor and mass-velocity distribution of the micro-jet from different groove angles and various wavelengths were presented. The numerical simulation results showed that the maximum velocity was sensitive to the change of groove angle and insensitive to the variety of groove wavelength, the ejecting factor and mass-velocity distribution changed remarkably with the variety of groove angle and wavelength. But when the groove wavelength was longer than 5 times the groove depth, the ejecting factor and mass-velocity distribution of the micro-jet maintained invariance.

Keywords: micro-jet, groove defects, shock loading, ejecting factor, Euler hydrodynamic method.
\end{abstract}

\section{Introduction}

As a shock induced surface effect, the ejection was known as the amount of material traveling faster than the free surface, when the shock wave reflected from it. There were many possible sources that might contribute to the formation of ejecta: machine marks, grain boundaries, inclusions, material voids, and damaged layers (Sorenson et al. [1]). 
It was Walsh et al. [2] who first observed the ejecting phenomenon in 1953. From then on, a lot of experimental and analytical research was carried out. Because of the multiformity of the ejecta sources and the complexity of the effecting factors, by now experiment and simulation were the main ways of research. Mass ejection experiments indicated that the micro-jet from a defect on the specimen surface was the main source of ejecta when surface melting didn't occur (Asay [3]). The ejection experiment conducted on a groove specimen showed that the groove angle affected the maximum ejecting velocity and total ejecting mass (Asay [4]). Mass ejection experiments conducted on Sn indicated that the surface roughness had great effect on the mass ejection (Zellner et al. [5]). The MD simulation result showed that the average velocity of the ejecting particles increased with the groove angle, and the amount of ejecting particles decreased as the groove angle increased (Jun et al. [6]). The SPH simulation result indicated that the ejecting factor reached its maximum as the half groove angle was equal to $45^{\circ}$, and the maximum velocity of ejecta showed a linear reduction with the increase of groove angle (Pei et al. [7]). The experimental and numerical results indicated that the free surface defect was an important source of ejecta, but some of the results weren't consistent. Therefore, the defect induced ejection needed to be investigated thoroughly.

There were two kinds of specimens used in the mass ejection experiment. One was a machined metal surface with natural defects of a quasi-seriate $\mathrm{V}$-groove. The other was specimens with artificial defects of a parallel V-groove, which was used to study the groove angle effect in some ejecting experiments. The wavelength and depth of the two kinds of V-groove were different. Those might contribute to the diverse trends in the experimental and numerical simulation result. A Euler code was used in simulating the two kinds of mass ejection models. When the groove angle and the wavelength changed, the maximum ejecting velocity and the mass distribution of a micro-jet were focused on.

\section{Computational method}

An elastic plastic hydrodynamic L-R type two steps Euler method Meph was applied in simulating the micro-ejection from the defect of the free surface. Meph was appropriated to simulate the high velocity impact and large deformation problems (Qijing et al. [8, 9]). A simplified equation of state for condensed medium, and an elastic-perfectly plastic constitutive model were used (see equations (1) and (2)). The material parameters used in the simulation are listed in table 1 .

Equation of state:

$$
p=C_{0}{ }^{2}\left(\rho-\rho_{0}\right)+(\gamma-1) \rho e
$$

Here, the initial density and sound velocity are $\rho_{0}$ and $C_{0}$, the constant of material is $\gamma$, the continuum density and specific internal energy are $\rho$ and $e$. 
Constitutive relationship:

$$
s_{x x}^{2}+s_{y y}^{2}+s_{z z}^{2}+2\left(s_{x y}^{2}+s_{y z}^{2}+s_{z x}^{2}\right) \leq \frac{2}{3}\left(Y_{0}\right)^{2}
$$

where $s_{i j}$ is the deviatoric stress and $Y_{0}$ is the yield strength.

Table 1: $\quad$ Material parameter used in the simulation.

\begin{tabular}{|c|c|c|c|c|}
\hline & $\rho_{0}\left(\mathrm{~g} / \mathrm{cm}^{3}\right)$ & $C_{0}(\mathrm{~km} / \mathrm{s})$ & $\gamma$ & $Y_{0}(\mathrm{GPa})$ \\
\hline $\mathrm{Al}$ & 2.71 & 5.38 & 3.03 & 0.29 \\
\hline
\end{tabular}

\section{Computational model}

There were two kinds of specimens using in the mass ejection experiment: (a) a machined metal surface with natural defects of a quasi-seriate V-groove; (b) in order to study the groove angle effect, specimens with artificial defects of a parallel V-groove were used in some ejecting experiments (see fig. 1). The study was focused on the maximum ejecting velocity and the mass distribution of the micro-jet, when the groove angle and the groove wavelength changed.

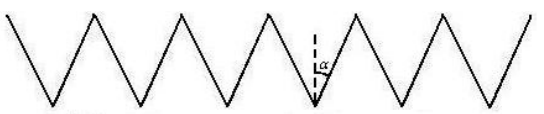

(a) Machined metal surface

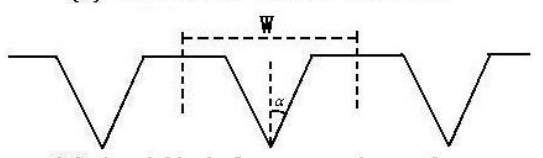

(b) Artificial grooved surface

Figure 1: Idealized defects on two kinds of surfaces.

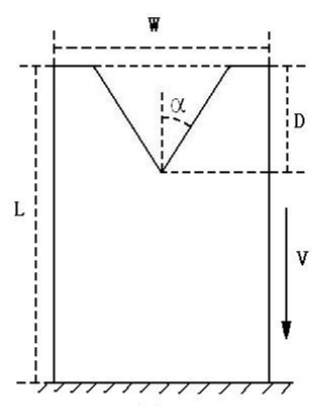

(a)

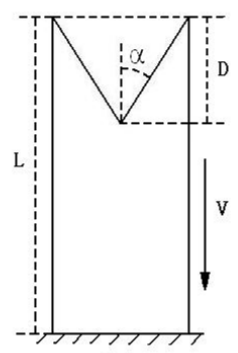

(b)

Figure 2: Numerical simulation model. 
The specimens used in the experiment were conducted on aluminum with artificial defects of a parallel V-groove (Asay [4]). The groove depth was 55um, the groove wavelength was $130 \mathrm{um}$ and the half groove angle varied from $15^{\circ}$ to $45^{\circ}$. Two kinds of models were used in the numerical simulation (see fig. 2). In model (a), the groove depth was $55 \mathrm{um}$, the groove wavelength was $130 \mathrm{um}$, the half groove angle varied from $15^{\circ}$ to $50^{\circ}$ and the height of model was $200 \mathrm{um}$. In model (b), the groove depth and the height of model were same as model a, the half groove angle varied from $15^{\circ}$ to $75^{\circ}$ and the groove wavelength varied with the angle.

\section{Simulation result}

\subsection{The micro-jetting forming process}

At the beginning, model (a) impacted the rigid boundary at $1.5 \mathrm{~km} / \mathrm{s}$, producing an Al shock pressure of about 30 GPa (fig. 3(a)). When the shock wave arrived at the bottom of groove, the rarefaction wave that reflected from the groove sidewall accelerated the material of the groove bottom (figs 3(b) and 4(a)). Groove sidewalls collapsed under the combined effect of shock wave and rarefaction wave; the fragmentation impacted on the symmetry axis. Under the high pressure of collision, a high speed jet formed (fig. 4(b)).
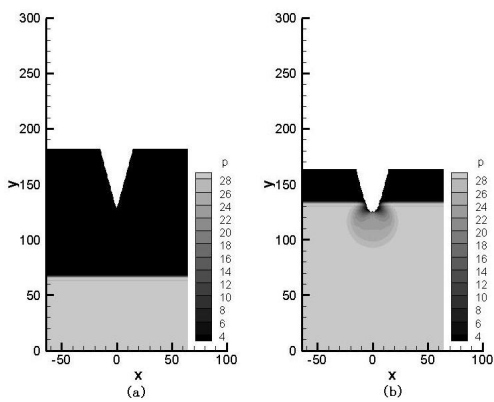

Figure 3: Pressure isoline image.
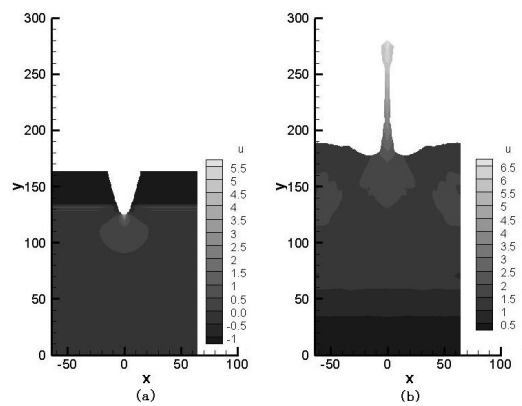

Figure 4: Velocity isoline image. 


\subsection{Effect of groove angle}

The maximum velocity of different angle grooves was given (see fig. 5). Where $\mathrm{V}_{\max }$ was the maximum velocity of micro-jetting, $\mathrm{V}_{\mathrm{fs}}$ was the free surface velocity. The simulation result of two kinds of model showed that the maximum velocity was fitting well with the experiment data. The maximum velocity decreased linearly as the groove angle increased. The maximum velocity was insensitive to the change of groove wavelength.

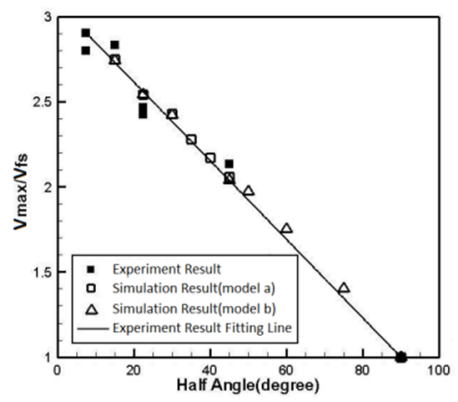

Figure 5: Maximum velocity of different angle grooves.

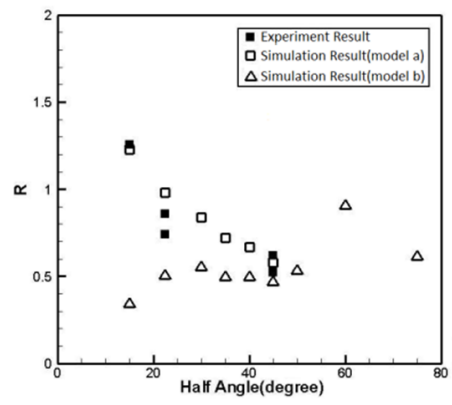

Figure 6: Ejecting factor of different angle grooves.

The ejecting factor (the ejecting mass divided by the product of groove volume and initial material density) simulation result of model (a) fitted well with the experiment data (as shown in fig. 6). The simulation result of model (a) and experiment data indicated that the ejecting factor decreased as the groove angle increased, when the groove wavelength was fixed. For model (b), the ejecting factor wasn't monotone, the maximum of ejecting factor was acquired when the half groove angle was about $60^{\circ}$.

Compared with model (a) whose groove wavelength was fixed, the simulation result of small groove angle cases in model (b) was slimmer. That meant for the same groove angle, the longer the groove wavelength, the more the ejecting mass.

The mass and velocity distribution curves of the two kinds of models are given in fig. 7. Where $\mathrm{V}$ was the micro-jetting velocity, $\mathrm{V}_{\mathrm{fs}}$ was the free surface velocity. 
Y-axis was the ejecta mass, whose non-dimensional velocity was more than the corresponding abscissa value, divided by the total ejecting mass.
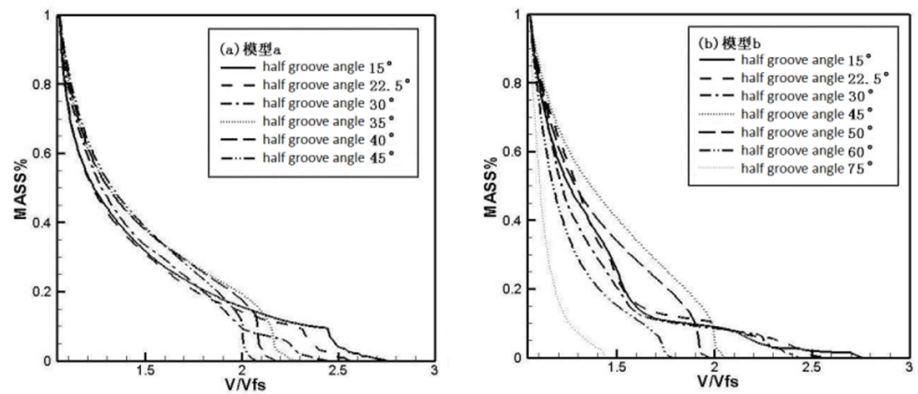

Figure 7: $\quad \mathrm{M}-\mathrm{V}$ distribution curve for ejection of different groove angles.

The simulation results of model (a) showed that the smaller the groove angle, the more the ratio of micro-jet tail mass (whose velocity was less than 1.1 times the free surface velocity) was to the total jet mass. The simulation results of model (b) indicated that the bigger the groove angle, the less the ratio of micro-jet tail mass to total jet mass, when the groove angle was less than $45^{\circ}$; the smaller groove angle, the less the ratio of micro-jet tail mass to total jet mass, when the groove angle was bigger than $45^{\circ}$. For models (a) and (b) the smaller groove angle, the faster the maximum velocity of micro-jetting.

\subsection{Effect of groove wavelength}

The ejecting factor of different groove wavelength was given in fig. 8, DIS was non-dimensional distance (wavelength divided by groove depth). It was concluded that the smaller the groove angle, the bigger the ejecting factor, when the groove wavelength was fixed. For the three cases in fig. 8, when the groove wavelength was smaller than 4 times the groove depth, the ejecting factor increased fast with the increase of wavelength; when the groove wavelength was bigger than 4 times but less than 5 times the groove depth, the ejecting factor increased slowly with

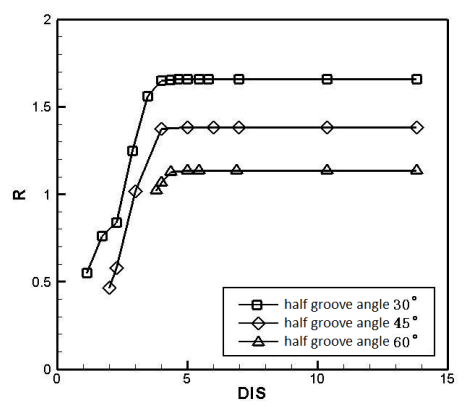

Figure 8: Ejection factor of different groove wavelengths. 

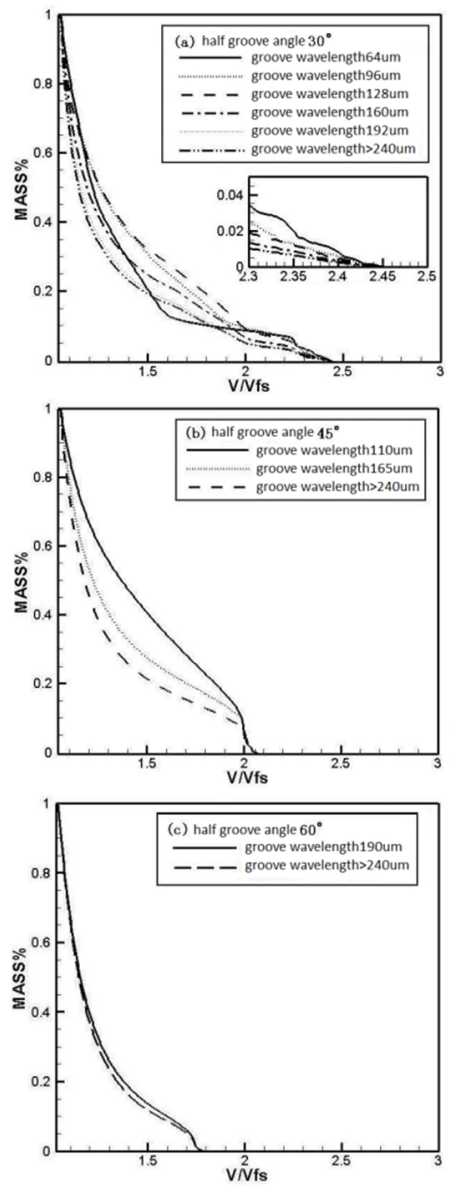

Figure 9: $\quad \mathrm{M}-\mathrm{V}$ distribution curve for ejection of different groove wavelengths.

the increase of wavelength; when the groove wavelength was bigger than 5 times the groove depth, the total ejecting mass or the ejecting factor remained constant.

The statistical result in fig. 9, the $\mathrm{M}-\mathrm{V}$ distribution curve of different groove wavelengths showed that the velocity of the micro-jet head was insensitive to the change of groove wavelength. But the ratio of micro-jet head mass to total jet mass decreased with the increase of groove wavelength. The ratio of micro-jet tail mass to total jet mass increased with the increasing of the groove wavelength. The M$\mathrm{V}$ distribution curve of ejecta changed very minimally when the groove wavelength was bigger than 4.4 times the groove depth. The total ejecting mass remained constant, and the $\mathrm{M}-\mathrm{V}$ distribution curve was invariable, when the groove wavelength was bigger than 5 times the groove depth. 


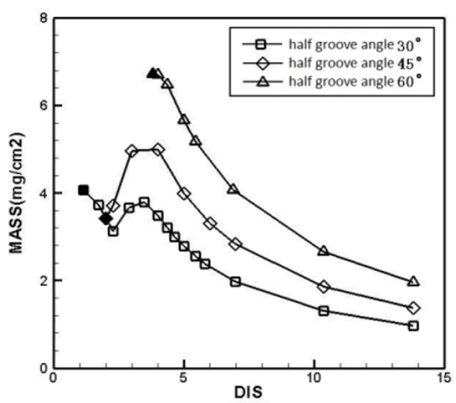

Figure 10: Ejecta mass of different groove wavelengths.

In the ejection experiment, Asay foil or quartz was usually used to obtain the ejecta mass from the unit surface of the specimen. In order to study the effect of groove wavelength on the ejecting mass from the unit surface, the ejecting mass of different groove wavelengths is given in fig. 10. The Y-axis was the total mass of micro-jet per square centimeter from the specimen surface. The hollow square, diamond and triangle represented the simulation results of $60^{\circ}, 45^{\circ}$ and $30^{\circ} \mathrm{V}$ grooves respectively. The solid one was the corresponding experiment result.

It is shown in fig. 10, when the groove wavelength was less than 5 times the groove depth, the ejecting factor increased, and the number of grooves in the unit surface decreased with the increasing of groove wavelength. Therefore, the ejecting mass from the unit specimen surface was non-monotone with the increase of groove wavelength, when the groove wavelength was less than 5 times the groove depth. When the groove wavelength was more than 5 times the groove depth, the ejecting factors remained constant, but the number of grooves in the unit surface decreased, so the ejecting mass from the unit specimen surface decreased as the groove wavelength increased.

\section{Conclusion}

An elastic plastic hydrodynamics Euler code was applied to simulate the microjet, which formed from the grooves in the surface under shock loading. The study was focused on the velocity and the mass of the micro-jet, when the groove angle and the groove wavelength changed.

The simulation results showed that when the groove angle increased, the maximum velocity decreased linearly, the ejecting factor and mass-velocity distribution of the micro-jet were obviously changed. The maximum velocity was insensitive to the change of groove wavelength, but the ejecting factor and massvelocity distribution changed remarkably when the groove wavelength increased. When the groove wavelength was more than 5 times the groove depth, the ejecting factor and mass-velocity distribution of the micro-jet maintained invariant, and the ejecting mass from unit specimen surface was decreased with the groove wavelength increasing. 


\section{References}

[1] Sorenson, D. S., Minich, R. W., Romero, J. L., Tunnell, T. W. \& Malone, R. M., Ejecta Particle Size Distributions for Shock Loaded Sn and Al Metals. J Appl Phys, 92(10), pp. 5830-5836, 2002.

[2] Walsh, J. M., Shreffler, R. G. \& Willing, F. J., Limiting Conditions for Jet Formation in High Velocity Collisions. J Appl Phys, 24(3), pp. 349-359, 1953.

[3] Asay, J. R., A Model for Estimating the Effects of Surface Roughness on Mass Ejection from Shocked Materials. SAND78-1256. Sandia Laboratories, 1978.

[4] Asay, J. R., Material Ejection from Shock-Loaded Free Surface of Aluminum and Lead. SAND76-0542. Sandia Laboratories, 1976.

[5] Zellner, M. B., Grover, M., Hammerberg, J. E., Hixson, R. S., Iverson, A. J., Macrum, G. S., Morley, K. B., Obst, A. W., Olson, R. T., Payton, J. R., Rigg, P. A., Routley, N., Stevens, G. D., Turley, W. D., Veeser, L. \& Buttler, W. T., Effects of shock-breakout pressure on ejection of micron-scale material from shocked tin surfaces. J Appl Phys, 102(013522), 2007.

[6] Jun C., Fuqian J. \& Jinglin Z., Molecular dynamics simulation of micro particle ejection from a shock-impacted metal surface. Acta Physica Sinica, 51(10), pp. 2386-2392, 2002.

[7] Pei W., Jianli S., \& Chengsen Q., Study of groove angle effect on micro-jet from shocked metal surface. Acta Physica Sinica, 61(23), 234701, 2012.

[8] Qijing F., Pengcheng H., Yihong H., Eulerian numerical simulation of a shaped charge. Explosion and Shock Waves, 28(2), pp. 138-143, 2008.

[9] Qijing F., Xianlin C., Pengcheng H., Fengguo Z., Shudao Z., Quanmin L. \& Haibo G., The simulating method study and application of well perforator operating process. Chinese Journal of Computational Physics, 26(6), pp. 887$891,2009$. 